Article

\title{
Skeletal Muscle Depletion Predicts the Prognosis of Patients with Hepatocellular Carcinoma Treated with Sorafenib
}

\author{
Kenji Imai *, Koji Takai, Tatsunori Hanai, Takayasu Ideta, Tsuneyuki Miyazaki, \\ Takahiro Kochi, Atsushi Suetsugu, Makoto Shiraki and Masahito Shimizu \\ Department of Gastroenterology/Internal Medicine, Gifu University Graduate School of Medicine, \\ 1-1 Yanagido, Gifu 501-1194, Japan; E-Mails: koz@gifu-u.ac.jp (K.T.); hanai0606@yahoo.co.jp (T.H.); \\ taka.mailbox.789@gmail.com (T.I.); tsunemiyazaking@yahoo.co.jp (T.M.); kottii924@gmail.com (T.K.); \\ asue327@yahoo.co.jp (A.S.); mshiraki-gif@umin.ac.jp (M.S.); shimim-gif@umin.ac.jp (M.S.) \\ * Author to whom correspondence should be addressed; E-Mail: ikenji@gifu-u.ac.jp; \\ Tel.: +81-58-230-6308; Fax: +81-58-230-6310.
}

Academic Editor: Tatsuo Kanda

Received: 3 February 2015 / Accepted: 22 April 2015 / Published: 28 April 2015

\begin{abstract}
The aim of this study was to determine whether skeletal muscle depletion predicts the prognosis of patients with hepatocellular carcinoma (HCC) that is being treated with sorafenib. We evaluated 40 consecutive HCC patients who received sorafenib treatment. The skeletal muscle cross-sectional area was measured by computed tomography at the third lumbar vertebra (L3), from which the L3 skeletal muscle index (L3 SMI) was obtained. The factors contributing to overall survival, sorafenib dose reduction, and discontinuation of sorafenib were analyzed using the Cox proportional hazards model. L3 SMI $(p=0.020)$ and $\log (\alpha$-fetoprotein (AFP) $(p=0.010)$ were identified as independent prognostic factors in HCC patients treated with sorafenib. The initial dose of sorafenib $(p=0.008)$ was an independent risk factor for sorafenib dose reduction, and log (AFP) $(p=0.008)$ was the only significant risk factor for the discontinuation of this drug. L3 SMI was not a risk factor for either dose reduction $(p=0.423)$ or the discontinuation $(p=0.132)$ of sorafenib. A multiple linear regression analysis determined the following relationship between skeletal muscle mass (assessed as L3 SMI) and the explanatory factors: L3 SMI $=-0.1896 \times($ Age $)-10.3441 \times($ Child-Pugh score $)-9.3922 \times(\log ($ AFP $))+$ $1.6139 \times(\log (\mathrm{AFP})) \times($ Child-Pugh score $)+112.9166$. Skeletal muscle depletion is inversely associated with age, Child-Pugh score, and log (AFP). Moreover, it is an independent prognostic factor for HCC patients treated with sorafenib.
\end{abstract}


Keywords: hepatocellular carcinoma; skeletal muscle depletion; sarcopenia; prognostic factor; sorafenib

\section{Introduction}

Hepatocellular carcinoma (HCC), which usually develops in the cirrhotic liver, is one of the most common malignancies worldwide [1,2]. It is essential to be able to predict the patient's prognosis in order to select the most adequate treatments for malignancies such as HCC. However, outcome prediction is extremely complicated for HCC because the liver functional reserve influences prognosis, sometimes to a greater extent than does the progression of HCC itself [3]. Further, the estimation of tumor stage alone is insufficient for predicting the prognosis of HCC since this malignancy very frequently recurs after curative treatment [4]. In order to predict the prognosis of HCC more precisely, several prognostic staging systems have been developed, such as the Barcelona Clinic Liver Cancer (BCLC) [5], Cancer of the Liver Italian Program (CLIP) [6], and Japan Integrated Staging (JIS) [7] systems. These staging systems are suitable for predicting HCC prognosis because they incorporate both tumor stages and liver functional reserves. However, it is also vital to continually make efforts to identify additional useful and simple factors that predict the prognosis of HCC.

Skeletal muscle depletion, or sarcopenia, was initially defined as the loss of skeletal muscle mass that occurs with aging [8]. Skeletal muscle depletion has been critically implicated in a variety of pathological conditions, such as malnutrition, advanced organ failure, and inflammatory disease [9]. It has also been identified as a poor prognostic factor for various types of human malignancies [10-13]. Particularly, cases of liver cirrhosis and HCC are frequently complicated by sarcopenia, which is associated with a poor prognosis for these diseases [14-17]. In a study of 217 consecutive patients with all stages of HCC, we found a strong relationship between skeletal muscle depletion and poor prognosis [16]. These previous findings, including our own data, suggest that skeletal muscle mass could be a useful and objective indicator for predicting the prognosis of patients with HCC.

Sorafenib (Nexavar, Bayer HealthCare Pharmaceuticals, Leverkusen, Germany) is the first orally active multi-kinase inhibitor that has been confirmed to be efficacious against advanced HCC $[18,19]$. Sorafenib has become the standard first-line treatment for patients with advanced HCC, but it sometimes causes severe side effects, such as hand-foot syndrome and liver dysfunction, which may limit the patient's ability to continue full-dose treatment [20,21]. A recent clinical trial showed that sarcopenia predicts early dose-limiting toxicities in patients who have advanced HCC with Child-Pugh A liver cirrhosis [20]. Therefore, we hypothesized that the poor prognosis of patients with sarcopenia and HCC might be caused by early dose reductions or the discontinuation of sorafenib due to its increased toxicity.

In the present study, we used computed tomography (CT) to measure the skeletal muscle cross-sectional areas of patients with HCC just before they began to receive treatment with sorafenib. Further, we designed a retrospective analysis to identify factors, including skeletal muscle mass, that could influence the overall survival of consecutive HCC patients treated with sorafenib. Finally, we 
have discussed the mechanisms by which skeletal muscle depletion affects mortality in HCC and the actions that should be taken to prevent sarcopenia in HCC patients treated with sorafenib

\section{Results}

\subsection{Baseline Characteristics and Laboratory Data}

The baseline characteristics and laboratory data of the 40 patients ( 34 men and 6 women; median age, 67 years) are shown in Table 1. The median L3 skeletal muscle index (SMI) was $41.9 \mathrm{~cm}^{2} / \mathrm{m}^{2}$ (range, 24.9-64.5 $\mathrm{cm}^{2} / \mathrm{m}^{2}$ ), and the median duration of follow-up was 12.2 months (range, 0.4-33.7 months). Three patients had already started to receive sorafenib when the disease had been in an earlier stage (Stage 0). Thirty-nine patients had received other treatments before they began to take sorafenib. Twenty-nine patients received combination treatments with sorafenib, 19 of whom received transcatheter arterial chemoembolization (TACE), 4 of whom received radiation therapy, and 6 of whom received both of these treatments.

Table 1. Baseline demographic and clinical characteristics.

\begin{tabular}{cc}
\hline Variables & $\boldsymbol{n}=\mathbf{4 0}$ \\
\hline Sex (male/female) & $34 / 6$ \\
Age (years) & $67(27-86)$ \\
Etiology (HBV/HCV/other) & $13 / 20 / 7$ \\
Follow-up period (months) & $12.2(0.4-33.7)$ \\
BMI $\left(\mathrm{kg} / \mathrm{m}^{2}\right)$ & $21.7(16.5-30.1)$ \\
L3 SMI $\left(\mathrm{cm}^{2} / \mathrm{m}^{2}\right)$ & $41.9(24.9-64.5)$ \\
Child-Pugh score $(5 / 6)$ & $27 / 13$ \\
Albumin $(\mathrm{g} / \mathrm{dL})$ & $3.6(2.8-4.6)$ \\
ALT $(\mathrm{IU} / \mathrm{L})$ & $26.5(8-65)$ \\
$\mathrm{T}-\mathrm{Bil}(\mathrm{mg} / \mathrm{dL})$ & $0.8(0.4-3.5)$ \\
PLT $\left(\times 10^{4} / \mu \mathrm{L}\right)$ & $11.6(2.1-61.3)$ \\
$\mathrm{PT}(\%)$ & $89.5(66-120)$ \\
AFP $(\mathrm{ng} / \mathrm{dL})$ & $100.2(2.3-211,459.8)$ \\
PIVKA-II (mAU/mL) & $253(15-254,450)$ \\
Stage $(0 / \mathrm{II} / \mathrm{III} / \mathrm{IVA} / \mathrm{IVB})$ & $3 / 0 / 4 / 9 / 4 / 20$ \\
Prior treatment $(\mathrm{yes} / \mathrm{no})$ & $39 / 1$ \\
Combination treatment $(\mathrm{TACE} / \mathrm{RT} / \mathrm{TACE}+\mathrm{RT} / \mathrm{no})$ & $19 / 4 / 6 / 11$ \\
Initial dose of sorafenib per day (mg) $(100 / 200 / 400 / 800)$ & $1 / 13 / 24 / 2$ \\
\hline
\end{tabular}

Values are presented as median (range). $\mathrm{HBV}$, hepatitis B virus; $\mathrm{HCV}$, hepatitis $\mathrm{C}$ virus; $\mathrm{BMI}$, body mass index; L3 SMI, third lumbar vertebra skeletal muscle index; ALT, alanine aminotransferase; T-Bil, total bilirubin; PLT, platelet count; PT, Prothrombin time; AFP, $\alpha$-fetoprotein; PIVKA-II, protein induced by vitamin $\mathrm{K}$ absence or antagonists-II; TACE, transarterial chemoembolization; RT, radiation therapy.

\subsection{Effects of Aging on Skeletal Muscle Depletion}

In order to evaluate the influence of aging on skeletal muscle depletion, we performed a paired $t$-test of the 36 cases in which CT was performed more than twice during the trial. This analysis showed that L3 SMI declined by an average of $2.7 \mathrm{~cm}^{2} / \mathrm{m}^{2}$ at an interval of 155 days $(p<0.0001)$. 


\subsection{Possible Prognostic Factors for HCC Patients}

Of the 40 patients, 20 died during the study period. Overall survival rates at one, two, and three years were $71.7 \%, 31.7 \%$, and $21.2 \%$, respectively, and the median duration of overall survival was 19.5 months (Figure 1a). In regards to response rates, 0, 3, 9 and 28 of the enrolled patients were classified as having complete response $(0 \%)$, partial response $(8.0 \%)$, stable disease $(22.0 \%)$, and progressive disease $(70.0 \%)$, respectively.

a)

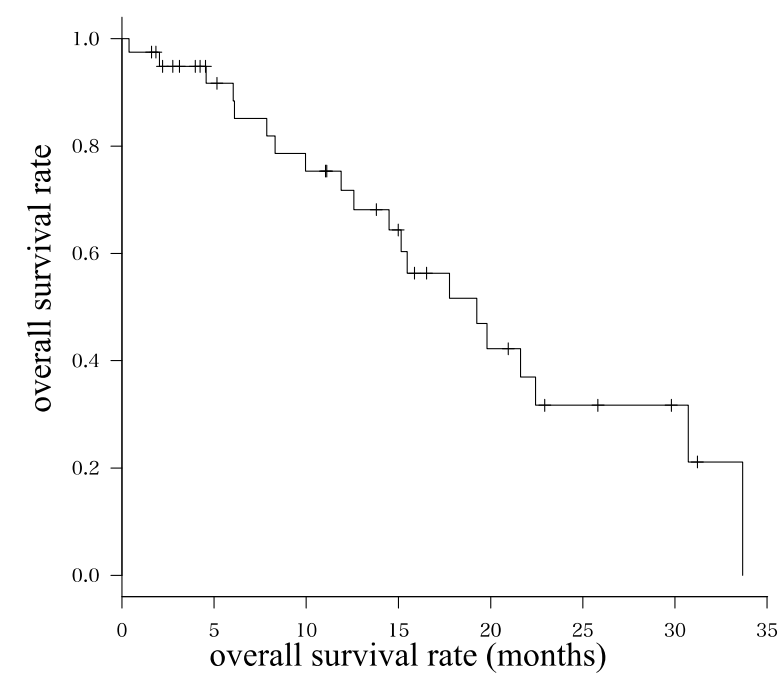

No. at Risk $\begin{array}{llllllll}40 & 29 & 23 & 16 & 9 & 5 & 3 & 0\end{array}$ b)

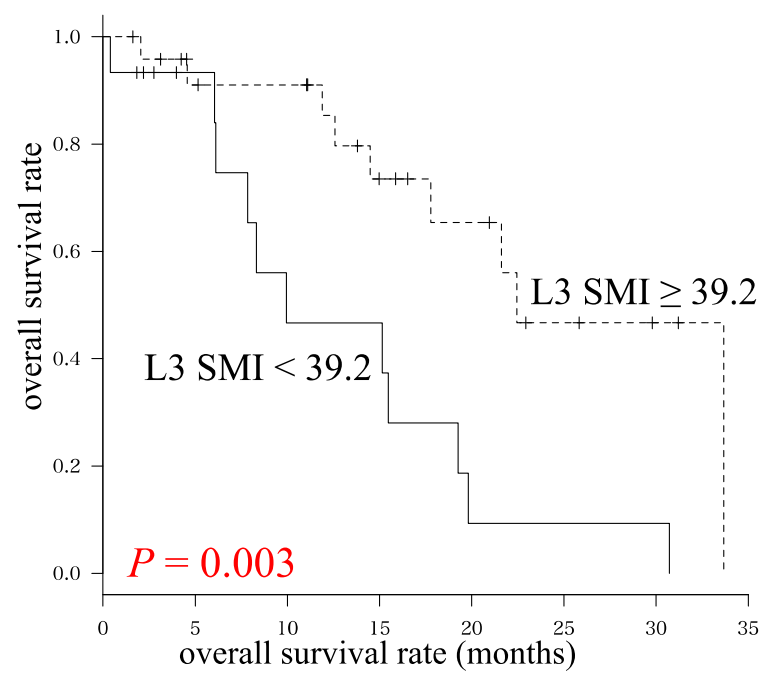

No. at Risk

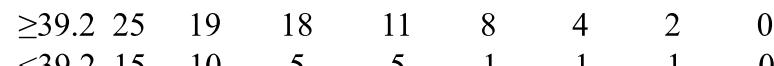

Figure 1. Kaplan-Meier curves for overall survival time in (a) all patients and (b) subgroups based on L3 skeletal muscle index (L3 SMI) $\left(<39.2\right.$ and $\left.\geq 39.2\left(\mathrm{~cm}^{2} / \mathrm{m}^{2}\right)\right)$.

We analyzed possible prognostic factors for HCC using the Cox proportional hazards model. Among the 10 variables listed in Table 2, L3 SMI $(p=0.020)$ and $\log (\alpha$-fetoprotein (AFP) $)(p=0.008)$ were significantly associated with the overall survival of patients with HCC in univariate analyses. Both L3 SMI $(p=0.020)$ and $\log$ (AFP) $(p=0.010)$ were also independent prognostic factors for HCC in our multivariate analysis. Furthermore, Kaplan-Meier analysis showed that overall survival was significantly shorter in patients with lower L3 SMI $\left(\leq 39.2 \mathrm{~cm}^{2} / \mathrm{m}^{2}, p=0.003\right.$, Figure $\left.1 \mathrm{~b}\right)$. The $39.2 \mathrm{~cm}^{2} / \mathrm{m}^{2}$ cut-off was selected to maximize the difference between the two survival curves, using a statistical analysis reported by Prado et al. [10]. In the present study, 15 patients had a L3 SMI of less than $39.2 \mathrm{~cm}^{2} / \mathrm{m}^{2}$ and 25 patients had an L3 SMI of more than $39.2 \mathrm{~cm}^{2} / \mathrm{m}^{2}$. 
Table 2. Univariate and multivariate analyses of possible risk factors for overall survival of hepatocellular carcinoma patients by the Cox proportional hazards model.

\begin{tabular}{ccccc}
\hline \multirow{2}{*}{ Variable } & \multicolumn{2}{c}{ Univariate Analysis } & \multicolumn{2}{c}{ Multivariate Analysis } \\
\cline { 2 - 5 } & HR (95\% CI) & $\boldsymbol{p}$ Value & HR (95\% CI) & $\boldsymbol{p}$ Value \\
\hline Age $($ years $)$ & $0.976(0.944-1.008)$ & 0.140 & & \\
BMI $\left(\mathrm{kg} / \mathrm{m}^{2}\right)$ & $0.863(0.715-1.042)$ & 0.127 & & \\
L3 SMI $\left(\mathrm{cm}^{2} / \mathrm{m}^{2}\right)$ & $0.904(0.830-0.984)$ & 0.020 & $0.909(0.836-0.985)$ & 0.020 \\
Child-Pugh score & $0.840(0.312-2.265)$ & 0.730 & & \\
Albumin $(\mathrm{g} / \mathrm{dL})$ & $0.556(0.180-1.722)$ & 0.309 & & \\
PLT $\left(\times 10^{4} / \mathrm{mL}\right)$ & $1.049(0.992-1.111)$ & 0.096 & & \\
$\log (\mathrm{AFP})$ & $1.793(1.162-2.767)$ & 0.008 & $1.274(1.0580-1.534)$ & \\
$\log (\mathrm{PIVKA}-\mathrm{II})$ & $1.024(0.857-1.224)$ & 0.794 & & \\
Stage (IVB $v s$. others) & $1.062(0.407-2.770)$ & 0.902 & & \\
Combination therapy (yes $v s$. no) & $0.533(0.188-1.509)$ & 0.236 & & \\
\hline
\end{tabular}

BMI, body mass index; L3 SMI, third lumbar vertebra skeletal muscle index; PLT, platelet count; AFP, $\alpha$-fetoprotein; PIVKA-II, protein induced by vitamin $\mathrm{K}$ absence or antagonists-II.

\subsection{Possible Risk Factors for Sorafenib Dose Reduction or Discontinuation Due to Toxicity}

During this study, the dose of sorafenib was reduced for 32 patients. Doses were reduced because of hand-foot syndrome in 6 patients, liver dysfunction in 5 patients, the progression of liver cirrhosis in 4 patients, deteriorating general condition in 4 patients, appetite loss in 3 patients, diarrhea in 2 patients, and assorted other reasons in the remaining 8 patients. As shown in Table 3, only the initial dose of sorafenib $(p=0.008)$ was significantly associated with sorafenib dose reduction.

Table 3. Univariate and multivariate analyses of possible risk factors for dose reduction of sorafenib due to its toxicity by Cox proportional hazards model.

\begin{tabular}{ccc}
\hline \multirow{2}{*}{ Variable } & \multicolumn{2}{c}{ Univariate Analysis } \\
\cline { 2 - 3 } & HR $(\mathbf{9 5 \%}$ CI) & $\boldsymbol{p}$ Value \\
\hline Age (years) & $0.980(0.954-1.007)$ & 0.143 \\
BMI $\left(\mathrm{kg} / \mathrm{m}^{2}\right)$ & $0.983(0.864-1.119)$ & 0.797 \\
L3 SMI & $0.979(0.928-1.032)$ & 0.423 \\
Child-Pugh score & $0.844(0.394-1.807)$ & 0.662 \\
Albumin (g/dL) & $0.789(0.338-1.840)$ & 0.583 \\
PLT $\left(\times 10^{4} / \mathrm{mL}\right)$ & $1.011(0.975-1.049)$ & 0.554 \\
PT $(\%)$ & $0.995(0.971-1.019)$ & 0.673 \\
T-Bil (mg/dL) & $1.696(0.782-3.681)$ & 0.181 \\
$\log (\mathrm{AFP})$ & $1.311(0.980-1.753)$ & 0.069 \\
$\log$ (PIVKA-II) & $1.010(0.891-1.145)$ & 0.876 \\
Stage (IVB vs. others) & $1.560(0.738-3.295)$ & 0.244 \\
Initial dose of sorafenib (mg) & $1.005(1.001-1.009)$ & 0.008 \\
\hline
\end{tabular}

BMI, body mass index; L3 SMI, third lumbar vertebra skeletal muscle index; PLT, platelet count; PT, Prothrombin time; T-Bil, total bilirubin; AFP, $\alpha$-fetoprotein; PIVKA-II, protein induced by vitamin K absence or antagonists-II. 
With respect to drug withdrawal, 28 patients were forced to discontinue sorafenib during this study. Ten patients discontinued sorafenib because of the deterioration of their general condition, 7 because of the progression of liver cirrhosis, 4 because of hand-foot syndrome, 3 because of disease progression, and the remaining 4 because of other factors. Among the variables listed in Table 4, $\log$ (AFP) $(p=0.008)$ was the only significant risk factor for the discontinuation of sorafenib. Neither sorafenib dose reduction ( $p=0.423)$ nor the discontinuation of sorafenib $(p=0.132)$ was significantly associated with the value of L3 SMI (Tables 3 and 4).

Table 4. Univariate and multivariate analyses of possible risk factors for discontinuation of sorafenib due to its toxicity by Cox proportional hazards model.

\begin{tabular}{ccc}
\hline \multirow{2}{*}{ Variable } & \multicolumn{2}{c}{ Univariate Analysis } \\
\cline { 2 - 3 } & HR (95\% CI) & $\boldsymbol{p}$ Value \\
\hline Age $($ years $)$ & $0.975(0.945-1.006)$ & 0.108 \\
BMI $\left(\mathrm{kg} / \mathrm{m}^{2}\right)$ & $0.927(0.802-1.072)$ & 0.307 \\
L3 SMI & $0.951(0.891-1.015)$ & 0.132 \\
Child-Pugh score & $0.987(0.448-2.176)$ & 0.974 \\
Albumin $(\mathrm{g} / \mathrm{dL})$ & $0.743(0.297-1.860)$ & 0.526 \\
PLT $\left(\times 10^{4} / \mathrm{mL}\right)$ & $1.017(0.975-1.062)$ & 0.436 \\
PT $(\%)$ & $0.998(0.973-1.023)$ & 0.859 \\
T-Bil $(\mathrm{mg} / \mathrm{dL})$ & $1.530(0.803-2.914)$ & 0.196 \\
log (AFP) & $1.222(1.055-1.415)$ & 0.008 \\
log (PIVKA-II) & $1.044(0.904-1.204)$ & 0.560 \\
Stage (IVB vs. others) & $1.559(0.727-3.346)$ & 0.254 \\
Initial dose of sorafenib $(\mathrm{mg})$ & $1.004(0.999-1.008)$ & 0.077 \\
\hline
\end{tabular}

BMI, body mass index; L3 SMI, third lumbar vertebra skeletal muscle index; PLT, platelet count; PT, Prothrombin time; T-Bil, total bilirubin; AFP, $\alpha$-fetoprotein; PIVKA-II, protein induced by vitamin K absence or antagonists-II.

\subsection{Multiple Linear Regression Analysis of L3 SMI and Significant Explanatory Valuables}

Loss of skeletal muscle mass is primarily a consequence of aging and secondarily a consequence of pathological conditions, such as malnutrition and complications of malignancy [9]. Men and women have different absolute skeletal muscle masses and rates of skeletal muscle mass reduction with increasing age $[22,23]$. Prompted by these earlier findings, we conducted a multiple regression analysis to examine the relationships between L3 SMI and 4 explanatory variables: age, Child-Pugh score, log (AFP), and sex. At first, we included each explanatory variable and all two-way interactions between explanatory variables within the multiple regressions. Subsequently, we removed the term with the highest $p$-value one by one until all remaining terms were significant $(p<0.05)$. In other words, we employed a backwards variable selection procedure. As detailed in Table 5, three individual explanatory variables and the interaction between $\log$ (AFP) and Child-Pugh score remained significant. The final result of the multiple regression analysis was as follows (with an intercept of 112.9166):

$$
\begin{array}{rlrl}
\text { L3 SMI }= & -0.1896 & \times(\text { Age }) \\
& -10.3441 & & (\text { Child-Pugh score }) \\
& -9.3922 & \times(\log (\text { AFP })) \\
& +1.6139 & \times(\log (\text { AFP })) \times(\text { Child-Pugh score }) \\
& +112.9166 &
\end{array}
$$


Table 5. Multiple linear regression analysis with L3 skeletal muscle index (SMI) and significantly explanatory valuables including each other's interactions.

\begin{tabular}{ccccc}
\hline Variables & Std. Coefficient & Std. Error & $\boldsymbol{t}$ Value & $\boldsymbol{p}$ Value \\
\hline Intercept & 112.9166 & 20.9930 & 5.38 & $<0.0001$ \\
Age & -0.1896 & 0.0758 & -2.50 & 0.017 \\
Child-Pugh score & -10.3441 & 3.9395 & -2.63 & 0.013 \\
$\log$ (AFP) & -9.3922 & 3.6142 & -2.60 & 0.014 \\
$\log$ (AFP): Child-Pugh score & 1.6139 & 0.6657 & 2.42 & 0.021 \\
\hline
\end{tabular}

Multiple R-squared: 0.338, F-statistic: 4.46 on 4 and 35 degree of freedom, $p$ value $=0.00512$.

This regression formula indicated that the level of L3 SMI was inversely associated with aging, worsening liver functional reserve, and increasing AFP levels.

\section{Discussion}

The results of the present study provide the first clear evidence that L3 SMI, an indicator of skeletal muscle mass or sarcopenia, is an independent prognostic factor for patients who have HCC and are being treated with sorafenib. These findings agree with previous studies that have shown significant relationships between skeletal muscle depletion and poor prognosis in patients with various clinical stages of HCC [14-17].

In the present study, we also obtained a regression equation that can be used to estimate L3 SMI from three explanatory factors: age, Child-Pugh score, and log (AFP). It has previously been reported that these factors may be associated with loss of skeletal muscle mass $[9,22,23]$. This analysis indicates that L3 SMI decreases in correspondence with increasing age, worsening liver functional reserve, and elevated AFP levels in patients with HCC who receive sorafenib. The interaction effect between log (AFP) and Child-Pugh score was quite small and can be ignored because of the large differences between the coefficient of the interaction effect and the coefficients of the associated main effects. Indeed, the combined value of the log (AFP) term, the Child-Pugh score term, and the interaction term $(1.6139 \times(\log ($ AFP $)) \times($ Child-Pugh score $)-10.3441 \times($ Child-Pugh score $)-9.3922 \times(\log ($ AFP $)))$ was far less than 0 in all patients (ranging from -66.46 to -52.82 ). The regression equation could present the key to explaining the association between skeletal muscle depletion and poor prognosis because liver functional reserve and AFP levels are both well-known prognostic factors for HCC [3]. Moreover, L3 SMI was affected by ageing, which itself affects the prognosis of patients with most medical conditions, even though existing prognostic staging systems for HCC (such as BCLC, CLIP, and JIS) do not include an aging parameter [5-7]. Thus, the evaluation of skeletal muscle mass using CT imaging could present a useful alternative method of predicting the prognosis of patients with HCC.

A recent clinical study reported that sarcopenia predicts early dose-limiting sorafenib toxicities, which might be involved in dose reductions or the discontinuation of this drug [20]. Therefore, we initially speculated that the more favorable prognosis of HCC patients without skeletal muscle depletion was associated with the greater extent of continuous sorafenib treatment that they received. However, contrary to this expectation, the loss of L3 SMI did not affect dose reductions or the discontinuation of sorafenib in the present study. We consider this finding to be explained by the initial dose of sorafenib, which was comparatively small. Specifically, the dose was less than $400 \mathrm{mg}$ per day 
in most cases (95\%). These low doses might strengthen the patients' adherence to sorafenib and, consequently, improve the prognosis of these patients in the present study. On the other hand, it should be noted that the initial dose was a risk factor for dose reduction. Further, we found that $\log$ (AFP) was a risk factor for the discontinuation of sorafenib. Therefore, it is necessary to be especially careful when facing cases of sorafenib-treated HCC that involve initial doses of $800 \mathrm{mg}$ per day or high AFP levels. In such cases, adverse events that require dose reductions or discontinuation may occur with a high probability.

Importantly, the survival outcomes of the present study were quite favorable as compared with a previous phase III trial by Llovet et al. In the present and previous studies, the median overall survival times were 19.5 and 10.7 months, respectively, and the survival rates at 1 year were $71.7 \%$ and $44.0 \%$, respectively [18]. The positive outcomes in our study may result from several factors. First, sorafenib was introduced at earlier tumor stage in some cases. Indeed, in three cases, it was used before obvious evidence of recurrent lesion had emerged, although pathological assessments of the resected specimen revealed either vascular invasion or focal positivity. Second, a combination treatment using sorafenib was administered in 29 cases $(72.5 \%)$. It is also interesting that, among the patients who received combination treatment, six cases (20.6\%) showed increased levels of skeletal muscle mass during this study, whereas only one case $(9.1 \%)$ showed this phenomenon in the group of patients who were treated with sorafenib alone. Combined treatment with sorafenib and TACE has a strong scientific rationale because the ischemia that is induced by TACE results in local and systemic increases in vascular endothelial growth factor (VEGF), whereas sorafenib inhibits the activity of the VEGF receptor [24]. Several meta-analyses have shown a positive outcome in HCC patients treated with TACE and sorafenib, as compared with HCC patients treated with TACE alone [25-27]. Currently, several randomized, controlled phase III trials of the safety and efficacy of sorafenib-based combination treatment are ongoing. These basic and clinical studies may provide new evidence that combination treatment with sorafenib is a promising approach for improving the prognosis of patients with HCC. However, additional careful investigation is necessary to verify the safety and efficacy of this combination treatment.

As demonstrated in the present study, skeletal muscle depletion seems to be an inevitable consequence of aging. On the other hand, it is possible that the prevention of skeletal muscle depletion might be an effective strategy for improving the prognosis of HCC; this approach should be considered further. Two possible interventions could prevent sarcopenia: nutritional and exercise therapies. Both of these interventions have been shown to improve outcomes for cirrhotic patients [28,29]. In particular, oral supplementation with branched-chain amino acids (BCAA; leucine, isoleucine and valine) is an effective nutritional therapy that can ameliorate protein energy malnutrition and improve event-free survival in cirrhotic patients [30-32]. BCAA supplementation is one of the most promising methods because it has been observed to improve the survival of sarcopenic patients with liver cirrhosis [33]. In a study of patients with alcoholic cirrhosis, Tsien $\mathrm{C}$ et al. demonstrated that impaired mTOR1 signaling and increased autophagy in skeletal muscle, which contribute progression of sarcopenia, are reversed by BCAA enriched with leucine [34]. Furthermore, the combination of a low intensity exercise and leucine-rich essential amino acid protein administration is effective for enhancing not only muscle strength, but also muscle mass and walking speed in sarcopenic women [35]. These reports suggest that BCAA supplementation, taking proper exercise, and the combination of these 
interventions might be effective for preventing sarcopenia. Future large-scale interventional studies should be conducted to determine whether nutritional and exercise therapies can prevent skeletal muscle depletion and possibly improve the prognosis of patients with HCC.

In summary, this study provides the first demonstration that L3 SMI, an indicator of skeletal muscle mass, is independently associated with poor prognosis among patients who have HCC and receive sorafenib treatment. In contrast, we found that L3 SMI was not a significant risk factor for dose reduction or the discontinuation of sorafenib. In addition, a multiple linear regression analysis suggested that L3 SMI was inversely associated with age, log (AFP), and Child-Pugh score, which indicated that these L3 SMI-correlated clinical features are also critical prognostic factors for patients with HCC. In conclusion, the evaluation of skeletal muscle mass using CT imaging could present a useful method of predicting the prognosis of patients with HCC, including those who are treated with sorafenib.

\section{Experimental Section}

\subsection{Patients, Treatment, and Follow-Up Strategy}

We evaluated 40 consecutive patients with HCC that was treated with sorafenib at our hospital between May 2009 and February 2014. Tumor stage was defined according to the staging system of the Liver Cancer Study Group of Japan [36]. HCC nodules were detected using imaging modalities including dynamic CT, dynamic magnetic resonance imaging (MRI), and abdominal arteriography. HCC was diagnosed on the basis of a typical hypervascular tumor stain on angiography and typical dynamic study findings of enhanced staining in the early phase and attenuation in the delayed phase. The objective of sorafenib introduction was determined according to the Clinical Practice Guidelines for HCC issued by the Japan Society of Hepatology [37]. Each patient's response was judged according to the Response Evaluation Criteria in Cancer of the Liver [38], which is an appropriate system for assessment of post-therapeutic response to sorafenib for HCC [39]. Overall survival was defined as the interval from the date of sorafenib introduction to the date of death, or until July 2014 for surviving patients. All study participants provided verbal informed consent, which was considered sufficient because this study followed an observational research design that did not require new human biological specimens, and instead relied only on preexisting materials. This study protocol was determined by following the Ethical Guidelines for Epidemiological Research (Ministry of Education, Culture, Sports, Science and Technology and Ministry of Health, Labour and Welfare in Japan, http://www.niph.go.jp/wadai/ekigakurinri/guidelines.pdf). The study design-including this consent procedure-was approved by the ethics committee of the Gifu University School of Medicine in 1 April 2014.

\subsection{Image Analysis of Skeletal Muscle Mass}

Skeletal muscle area was measured using a CT image that had been taken solely for the purpose of diagnosing HCC just before sorafenib was initiated. A transverse CT image at the third lumbar vertebra (L3) in the inferior direction was assessed as has been described previously [10]. The muscles in the L3 region (containing the psoas, erector spinae, quadratus lumborum, transversus abdominis, external 
and internal obliques, and rectus abdominis) were analyzed using SliceOmatic software version 5.0 (Tomovision, Montreal, QC, Canada), which enables specific tissue demarcation using Hounsfield unit (HU) thresholds. The muscles were quantified within a HU range of -29 to $+150 \mathrm{HU}$ [40], and tissue boundaries were manually corrected as needed. The cross-sectional areas of muscle $\left(\mathrm{cm}^{2}\right)$ at the L3 level were computed from each image and normalized by the square of the height $\left(\mathrm{m}^{2}\right)$ to obtain the L3 skeletal muscle index (L3 SMI, $\mathrm{cm}^{2} / \mathrm{m}^{2}$ ), which was used as an indicator of skeletal muscle mass [16]. The progression of skeletal muscle depletion was evaluated by comparing the values of L3 SMI for cases in which CT was performed more than twice during the trial.

\subsection{Statistical Analysis}

Overall survival was estimated using the Kaplan-Meier method, and differences between curves were evaluated using the log-rank test. Prognostic factors affecting overall survival were estimated using the Cox proportional hazards model. Parameters determined to be significant according to univariate analyses were then included in a multivariate analysis. Parameters affecting the interval from the date of sorafenib introduction to the date of dose reduction or discontinuation due to sorafenib toxicity were also estimated in the same manner. Statistical significance was defined as $p<0.05$. All statistical analyses were carried out using R version 3.1.1 (R Foundation for Statistical Computing, Vienna, Austria. URL: http://www.R-project.org/).

\section{Acknowledgments}

This work was supported, in part, by Grants-in-Aid from the Ministry of Education, Science, Sports and Culture of Japan (No. 22790638 and 25460988); Grant-in-Aid for the 3rd Term Comprehensive 10-Year Strategy for Cancer Control from the Ministry of Health, Labour, and Welfare of Japan; and Takeda Science Foundation. We thank Ms. Chiyoko Sano for her excellent technical assistance.

\section{Author Contributions}

Kenji Imai conceived and designed the study, performed statistical analysis, and wrote the manuscript. Kenji Imai, Koji Takai, Tatsunori Hanai, Takayasu Ideta, Tsuneyuki Miyazaki, Takahiro Kochi, Atsushi Suetsugu, and Makoto Shiraki enrolled the patients in this study and treated them. Masahito Shimizu helped and supervised to complete the manuscript. All authors read and approved the final manuscript.

\section{Conflicts of Interest}

The authors declare no conflict of interest.

\section{References}

1. El-Serag, H.B. Hepatocellular carcinoma: An epidemiologic view. J. Clin. Gastroenterol. 2002, 35, S72-S78.

2. Jemal, A.; Bray, F.; Center, M.M.; Ferlay, J.; Ward, E.; Forman, D. Global cancer statistics. CA Cancer J. Clin. 2011, 61, 69-90. 
3. Op den Winkel, M.; Nagel, D.; Sappl, J.; op den Winkel, P.; Lamerz, R.; Zech, C.J.; Straub, G.; Nickel, T.; Rentsch, M.; Stieber, P.; et al. Prognosis of patients with hepatocellular carcinoma. Validation and ranking of established staging-systems in a large western HCC-cohort. PLoS ONE 2012, 7, e45066.

4. Poon, R.T. Prevention of recurrence after resection of hepatocellular carcinoma: A daunting challenge. Hepatology 2011, 54, 757-759.

5. Llovet, J.M.; Bru, C.; Bruix, J. Prognosis of hepatocellular carcinoma: The BCLC staging classification. Semin. Liver Dis. 1999, 19, 329-338.

6. Japan Society of Hepatology. A new prognostic system for hepatocellular carcinoma: A retrospective study of 435 patients: The cancer of the liver italian program (CLIP) investigators. Hepatology 1998, 28, 751-755.

7. Kudo, M.; Chung, H.; Osaki, Y. Prognostic staging system for hepatocellular carcinoma (CLIP score): Its value and limitations, and a proposal for a new staging system, the Japan integrated staging score (JIS score). J. Gastroenterol. 2003, 38, 207-215.

8. Rosenberg, I.H. Sarcopenia: Origins and clinical relevance. J. Nutr. 1997, 127, S990-S991.

9. Cruz-Jentoft, A.J.; Baeyens, J.P.; Bauer, J.M.; Boirie, Y.; Cederholm, T.; Landi, F.; Martin, F.C.; Michel, J.P.; Rolland, Y.; Schneider, S.M.; et al. Sarcopenia: European consensus on definition and diagnosis: Report of the european working group on sarcopenia in older people. Age Ageing 2010, 39, 412-423.

10. Prado, C.M.; Lieffers, J.R.; McCargar, L.J.; Reiman, T.; Sawyer, M.B.; Martin, L.; Baracos, V.E. Prevalence and clinical implications of sarcopenic obesity in patients with solid tumours of the respiratory and gastrointestinal tracts: A population-based study. Lancet Oncol. 2008, 9, 629-635.

11. Tan, B.H.; Birdsell, L.A.; Martin, L.; Baracos, V.E.; Fearon, K.C. Sarcopenia in an overweight or obese patient is an adverse prognostic factor in pancreatic cancer. Clin. Cancer Res. 2009, 15, 6973-6979.

12. Sabel, M.S.; Lee, J.; Cai, S.; Englesbe, M.J.; Holcombe, S.; Wang, S. Sarcopenia as a prognostic factor among patients with stage III melanoma. Ann. Surg. Oncol. 2011, 18, 3579-3585.

13. Van Vledder, M.G.; Levolger, S.; Ayez, N.; Verhoef, C.; Tran, T.C.; Ijzermans, J.N. Body composition and outcome in patients undergoing resection of colorectal liver metastases. Br. J. Surg. 2012, 99, 550-557.

14. Dodson, R.M.; Firoozmand, A.; Hyder, O.; Tacher, V.; Cosgrove, D.P.; Bhagat, N.; Herman, J.M.; Wolfgang, C.L.; Geschwind, J.F.; Kamel, I.R.; et al. Impact of sarcopenia on outcomes following intra-arterial therapy of hepatic malignancies. J. Gastrointest. Surg. 2013, 17, 2123-2132.

15. Harimoto, N.; Shirabe, K.; Yamashita, Y.I.; Ikegami, T.; Yoshizumi, T.; Soejima, Y.; Ikeda, T.; Maehara, Y.; Nishie, A.; Yamanaka, T. Sarcopenia as a predictor of prognosis in patients following hepatectomy for hepatocellular carcinoma. Br. J. Surg. 2013, 100, 1523-1530.

16. Iritani, S.; Imai, K.; Takai, K.; Hanai, T.; Ideta, T.; Miyazaki, T.; Suetsugu, A.; Shiraki, M.; Shimizu, M.; Moriwaki, H. Skeletal muscle depletion is an independent prognostic factor for hepatocellular carcinoma. J. Gastroenterol. 2015, 50, 323-332.

17. Kaido, T.; Ogawa, K.; Fujimoto, Y.; Ogura, Y.; Hata, K.; Ito, T.; Tomiyama, K.; Yagi, S.; Mori, A.; Uemoto, S. Impact of sarcopenia on survival in patients undergoing living donor liver transplantation. Am. J. Transplant. 2013, 13, 1549-1556. 
18. Llovet, J.M.; Ricci, S.; Mazzaferro, V.; Hilgard, P.; Gane, E.; Blanc, J.F.; de Oliveira, A.C.; Santoro, A.; Raoul, J.L.; Forner, A.; et al. Sorafenib in advanced hepatocellular carcinoma. N. Engl. J. Med. 2008, 359, 378-390.

19. Wilhelm, S.; Carter, C.; Lynch, M.; Lowinger, T.; Dumas, J.; Smith, R.A.; Schwartz, B.; Simantov, R.; Kelley, S. Discovery and development of sorafenib: A multikinase inhibitor for treating cancer. Nat. Rev. Drug. Discov. 2006, 5, 835-844.

20. Mir, O.; Coriat, R.; Blanchet, B.; Durand, J.P.; Boudou-Rouquette, P.; Michels, J.; Ropert, S.; Vidal, M.; Pol, S.; Chaussade, S.; et al. Sarcopenia predicts early dose-limiting toxicities and pharmacokinetics of sorafenib in patients with hepatocellular carcinoma. PLoS ONE 2012, 7, e37563.

21. Huillard, O.; Boissier, E.; Blanchet, B.; Thomas-Schoemann, A.; Cessot, A.; Boudou-Rouquette, P.; Durand, J.P.; Coriat, R.; Giroux, J.; Alexandre, J.; et al. Drug safety evaluation of sorafenib for treatment of solid tumors: Consequences for the risk assessment and management of cancer patients. Expert Opin. Drug Saf. 2014, 13, 663-673.

22. Baumgartner, R.N.; Koehler, K.M.; Gallagher, D.; Romero, L.; Heymsfield, S.B.; Ross, R.R.; Garry, P.J.; Lindeman, R.D. Epidemiology of sarcopenia among the elderly in new mexico. Am. J. Epidemiol. 1998, 147, 755-763.

23. Sanada, K.; Miyachi, M.; Tanimoto, M.; Yamamoto, K.; Murakami, H.; Okumura, S.; Gando, Y.; Suzuki, K.; Tabata, I.; Higuchi, M. A cross-sectional study of sarcopenia in Japanese men and women: Reference values and association with cardiovascular risk factors. Eur. J. Appl. Physiol. 2010, 110, 57-65.

24. Weintraub, J.L.; Salem, R. Treatment of hepatocellular carcinoma combining sorafenib and transarterial locoregional therapy: State of the science. J. Vasc. Interv. Radiol. 2013, 24, 1123-1134.

25. Fu, Q.H.; Zhang, Q.; Bai, X.L.; Hu, Q.D.; Su, W.; Chen, Y.W.; Su, R.G.; Liang, T.B. Sorafenib enhances effects of transarterial chemoembolization for hepatocellular carcinoma: A systematic review and meta-analysis. J. Cancer Res. Clin. Oncol. 2014, 140, 1429-1440.

26. Yang, M.; Yuan, J.Q.; Bai, M.; Han, G.H. Transarterial chemoembolization combined with sorafenib for unresectable hepatocellular carcinoma: A systematic review and meta-analysis. Mol. Biol. Rep. 2014, 41, 6575-6582.

27. Zhang, L.; Hu, P.; Chen, X.; Bie, P. Transarterial chemoembolization (tace) plus sorafenib vs. tace for intermediate or advanced stage hepatocellular carcinoma: A meta-analysis. PLoS ONE 2014, 9 , e100305.

28. Jones, J.C.; Coombes, J.S.; Macdonald, G.A. Exercise capacity and muscle strength in patients with cirrhosis. Liver Transpl. 2012, 18, 146-151.

29. Plauth, M.; Cabre, E.; Riggio, O.; Assis-Camilo, M.; Pirlich, M.; Kondrup, J.; Ferenci, P.; Holm, E.; Vom Dahl, S.; Muller, M.J.; et al. Espen guidelines on enteral nutrition: Liver disease. Clin. Nutr. 2006, 25, 285-294.

30. Marchesini, G.; Bianchi, G.; Merli, M.; Amodio, P.; Panella, C.; Loguercio, C.; Rossi Fanelli, F.; Abbiati, R. Nutritional supplementation with branched-chain amino acids in advanced cirrhosis: A double-blind, randomized trial. Gastroenterology 2003, 124, 1792-1801. 
31. Moriwaki, H.; Shiraki, M.; Fukushima, H.; Shimizu, M.; Iwasa, J.; Naiki, T.; Nagaki, M. Long-term outcome of branched-chain amino acid treatment in patients with liver cirrhosis. Hepatol. Res. 2008, 38, S102-S106.

32. Muto, Y.; Sato, S.; Watanabe, A.; Moriwaki, H.; Suzuki, K.; Kato, A.; Kato, M.; Nakamura, T.; Higuchi, K.; Nishiguchi, S.; et al. Effects of oral branched-chain amino acid granules on event-free survival in patients with liver cirrhosis. Clin. Gastroenterol. Hepatol. 2005, 3, 705-713.

33. Hanai, T.; Shiraki, M.; Nishimura, K.; Ohnishi, S.; Imai, K.; Suetsugu, A.; Takai, K.; Shimizu, M.; Moriwaki, H. Sarcopenia impairs prognosis of patients with liver cirrhosis. Nutrition 2015, 31, 193-199.

34. Tsien, C.; Davuluri, G.; Singh, D.; Allawy, A.; Ten Have, G.A.; Thapaliya, S.; Schulze, J.M.; Barnes, D.; McCullough, A.J.; Engelen, M.P.; et al. Metabolic and molecular responses to leucine-enriched branched chain amino acid supplementation in the skeletal muscle of alcoholic cirrhosis. Hepatology 2015, doi:10.1002/hep.27717.

35. Kim, H.K.; Suzuki, T.; Saito, K.; Yoshida, H.; Kobayashi, H.; Kato, H.; Katayama, M. Effects of exercise and amino acid supplementation on body composition and physical function in community-dwelling elderly Japanese sarcopenic women: A randomized controlled trial. J. Am. Geriatr. Soc. 2012, 60, 16-23.

36. Japan Society of Hepatology. The general rules for the clinical and pathological study of primary liver cancer. Liver cancer study group of Japan. Jpn. J. Surg. 1989, 19, 98-129.

37. Japan Society of Hepatology. Clinical practice guidelines for hepatocellular carcinoma-The Japan society of hepatology 2009 update. Hepatol. Res. 2010, 40, S2-S144.

38. Kudo, M.; Kubo, S.; Takayasu, K.; Sakamoto, M.; Tanaka, M.; Ikai, I.; Furuse, J.; Nakamura, K.; Makuuchi, M. Response evaluation criteria in cancer of the liver (recicl) proposed by the liver cancer study group of Japan (2009 revised version). Hepatol. Res. 2010, 40, 686-692.

39. Arizumi, T.; Ueshima, K.; Takeda, H.; Osaki, Y.; Takita, M.; Inoue, T.; Kitai, S.; Yada, N.; Hagiwara, S.; Minami, Y.; et al. Comparison of systems for assessment of post-therapeutic response to sorafenib for hepatocellular carcinoma. J. Gastroenterol. 2014, 49, 1578-1587.

40. Mitsiopoulos, N.; Baumgartner, R.N.; Heymsfield, S.B.; Lyons, W.; Gallagher, D.; Ross, R. Cadaver validation of skeletal muscle measurement by magnetic resonance imaging and computerized tomography. J. Appl. Physiol. 1998, 85, 115-122.

(C) 2015 by the authors; licensee MDPI, Basel, Switzerland. This article is an open access article distributed under the terms and conditions of the Creative Commons Attribution license (http://creativecommons.org/licenses/by/4.0/). 